DALAT UNIVERSITY JOURNAL OF SCIENCE Volume 12, Issue 2, 2022 113-122

\title{
EULER CHARACTERISTIC OF TANGO BUNDLES
}

\author{
Nguyen Hong Cong ${ }^{a}$, Dang Tuan Hiep ${ }^{b^{*}}$, Nguyen Thi Mai Van \\ ${ }^{a}$ Asia Pacific College, Gia Lai, Vietnam \\ ${ }^{b}$ Faculty of Mathematics and Computer Science, Da Lat University, Lam Dong, Vietnam \\ ${ }^{c}$ Faculty of Mathematics and Statistics, Quy Nhon University, Binh Dinh, Vietnam \\ ${ }^{*}$ Corresponding author: Email: hiepdt@dlu.edu.vn
}

\section{Article history}

Received: July $26^{\text {th }}, 2021$

Received in revised form: October $1^{\text {st }}, 2021 \mid$ Accepted: October 12 $2^{\text {th }}, 2021$

Available online: January $27^{\text {th }}, 2022$

\begin{abstract}
We are interested in a vector bundle constructed by $\mathbf{T}$. The Tango bundle is an indecomposable vector bundle of rank $n-1$ on the complex projective space $\mathbb{P}^{n}$. In particular, we show that the Euler characteristic of the Tango bundle on $\mathbb{P}^{n}$ is equal to $2 n-1$.
\end{abstract}

Keywords: Euler characteristic; Tango bundle.

DOI:http://dx.doi.org/10.37569/DalatUniversity.12.2.956(2022)

Article type: (peer-reviewed) Full-length research article

Copyright $@ 2022$ The author(s)

Licensing: This article is licensed under a CC BY-NC 4.0 


\section{INTRODUCTION}

In this paper, we will use the following notations. Let $\mathbb{P}^{n}$ be the $n$-dimensional complex projective space. $\mathscr{O}_{\mathbb{P}^{n}}(m)$ is the line bundle (vector bundle of rank 1) on $\mathbb{P}^{n}$ associated with the divisor $m h$, where $h$ is the hyperplane class on $\mathbb{P}^{n}$ and $m$ is an integer. $T_{\mathbb{P} n}$ is the tangent bundle on $\mathbb{P}^{n}$. For a vector bundle $E$, we denote by $c(E)$ the total Chern class, by $\operatorname{ch}(E)$ the Chern character, and by $\operatorname{td}(E)$ the Todd class of $E$.

A vector bundle on $\mathbb{P}^{n}$ is said to be indecomposable if it is not a direct sum of vector bundles of smaller rank. It is difficult to construct indecomposable vector bundles of small rank on the projective space $\mathbb{P}^{n}$. In fact, the Hartshorne conjecture (see Hartshorne, 1979) asserts that we can not construct such bundles for large dimension $n$ and small rank. More precisely, every bundle of rank 2 on $\mathbb{P}^{n}$, where $n \geq 7$, splits as a direct sum of line bundles.

An interesting example of an indecomposable vector bundle on $\mathbb{P}^{n}$ was constructed by Tango (1976). Consider the following exact sequence of vector bundles

$$
0 \longrightarrow T_{\mathbb{P}^{n}}(-2) \longrightarrow F_{1} \longrightarrow E_{n} \longrightarrow 0,
$$

where $F_{1}$ is the trivial vector bundle of rank $\frac{1}{2} n(n+1)$ and $T_{\mathbb{P} n}(-2)$ is the vector bundle $T_{\mathbb{P} n} \otimes \mathscr{O}_{\mathbb{P}^{n}}(-2)$, and where $\mathscr{O}_{\mathbb{P}}(-2)$ is the line bundle on $\mathbb{P}^{n}$ with the first Chern class $c_{1}=-2 h$.

It was proved by Tango that $E_{n}$ is generated by its global sections and that $c_{n}\left(E_{n}\right)=$ 0 (see Tango, 1976). It follows that $E_{n}$ has a trivial vector bundle $F_{2}$ of rank $\frac{1}{2} n(n-1)-$ $n+1$ as a subbundle. Let $T_{n}$ be the quotient bundle of $E_{n}$ by $F_{2}$, then $T_{n}$ is a vector bundle of rank $n-1$. More precisely, the Tango bundle $T_{n}$ is determined by the following exact sequence

$$
0 \longrightarrow F_{2} \longrightarrow E_{n} \longrightarrow T_{n} \longrightarrow 0 \text {. }
$$

The geometric aspects of Tango bundles were studied by Jaczewski et al. (1986). The aim of this paper is to establish a general formula for the Euler characteristic of Tango bundles. Our main result is the following theorem.

Theorem 1. Let $n \geq 3$ be an integer. The Euler characteristic of the Tango bundle $T_{n}$ on $\mathbb{P}^{n}$ is equal to $2 n-1$.

Our approach is based on the Hirzebruch-Riemann-Roch theorem and the computation of Chern characters of Tango bundles and Todd classes of tangent bundles on projective spaces.

\section{PRELIMINARIES}

In this section, we review some basic notions, such as vector bundles, Chern classes, Chern characters, and Todd classes. All definitions and results in this section, 
along with the detail explanations and proofs, can be found in Eisenbud and Harris (2016), Fulton (1998), Dang (2014), Hirzebruch (1978), and Okonek et al. (1980).

\subsection{Vector bundles and Chern classes}

Let $X$ be a variety. A vector bundle of rank $n$ on $X$ is a variety $E$ together with a morphism $\pi: E \rightarrow X$, such that there is an open covering $\left\{U_{i}\right\}$ of $X$ satisfying the following conditions:

(i) For each $i \in I$, there is an isomorphism $\varphi_{i}: \pi^{-1}\left(U_{i}\right) \rightarrow U_{i} \times \mathbb{C}^{n}$ such that the following diagram commutes,

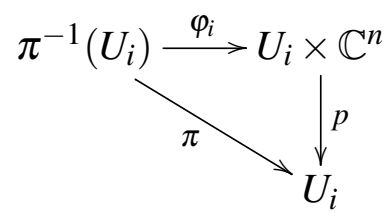

where $p: U_{i} \times \mathbb{C}^{n} \rightarrow U_{i}$ is the natural projection onto the first factor.

(ii) For each $i, j \in I$, there is an $n \times n$-matrix $\left(A_{i j}\right)$, whose entries are regular functions on $U_{i} \cap U_{j}$, such that the composition

$$
\psi_{i j}=\varphi_{j} \circ \varphi_{i}^{-1}:\left(U_{i} \cap U_{j}\right) \times \mathbb{C}^{n} \longrightarrow\left(U_{i} \cap U_{j}\right) \times \mathbb{C}^{n}
$$

is given by $\psi_{i j}(x, v)=\left(x, A_{i j}(x) v\right)$.

The variety $E$ is called the total space, but we often denote the entire vector bundle by the notation for the total space. The vector bundles of rank 1 are called line bundles.

Let $E$ be a vector bundle on a variety $X$. A section of $E$ over an open set $U \subseteq X$ is a morphism $s: U \rightarrow E$ such that $\pi \circ s$ is the identity on $U$. The set of sections of $E$ over $U$ is denoted by $\mathscr{E}(U)$. A section over $X$ is called global.

We say that a vector bundle is globally generated if it generated by its golbal sections. Let $X$ be a smooth variety of dimension $n$, and $E$ be a globally generated vector bundle of rank $r$ on $X$. Given a section $s \in \mathscr{E}(X)$ of $E$, one can show that the zero locus of $s$ is a cycle of codimension $r$ and its cycle class does not depend on the choice of $s$. We denote

$$
Z(s)=\{x \in X \mid s(x)=0\} .
$$

The top Chern class of $E$ is defined to be

$$
c_{r}(E)=[Z(s)]
$$

More generally, given a collection of $r-k+1$ sections $s_{1}, \ldots, s_{r-k+1} \in \mathscr{E}(X)$ of $E$, one can show that the locus where the $s_{i}$ fail to be independent is a cycle of codimension $k$ and its cycle class does not depend on the choice of $s_{1}, \ldots, s_{r-k+1}$. We denote

$$
Z\left(s_{1} \wedge \cdots \wedge s_{r-k+1}\right)=\left\{x \in X \mid s_{1}(x), \ldots, s_{r-k+1}(x) \text { are linearly dependent }\right\} .
$$


The $k$-th Chern class of $E$ is defined to be

$$
c_{k}(E)=\left[Z\left(s_{1} \wedge \cdots \wedge s_{r-k+1}\right)\right] .
$$

The total Chern class of $E$ is the sum

$$
c(E)=1+c_{1}(E)+\cdots+c_{r}(E) .
$$

Here are the basic properties of Chern classes:

(1) $c_{0}(E)=1$.

(2) $c_{i}(E)=0$ whenever $i>\operatorname{rank}(E)$.

(3) If $E^{\vee}=\operatorname{Hom}(E, \mathbb{C})$ is the dual of $E$, then

$$
c_{i}\left(E^{\vee}\right)=(-1)^{i} c_{i}(E) \text { for all } i .
$$

(4) If $E$ and $F$ are two vector bundles, then

$$
c(E \oplus F)=c(E) c(F) .
$$

Equivalently, we have

$$
c_{k}(E \oplus F)=\sum_{i=0}^{k} c_{i}(E) c_{k-i}(F) .
$$

\subsection{Splitting principle}

The splitting principle is a useful technique for reducing questions concerning vector bundles to questions concerning line bundles.

Let $E$ be a vector bundle of rank $r$ on a variety $X$. The splitting principle says that we can regard the Chern classes of $E$ as the elementary symmetric polynomials in $r$ variables $\alpha_{i}$ for all $i=1, \ldots, r$, which are called the Chern roots of $E$. More precisely, we have

$$
\begin{aligned}
c_{0}(E) & =1, \\
c_{1}(E) & =\sum_{1 \leq i \leq r} \alpha_{i}, \\
c_{2}(E) & =\sum_{1 \leq i<j \leq r} \alpha_{i} \alpha_{j}, \\
\vdots & \\
c_{r}(E) & =\alpha_{1} \alpha_{2} \ldots \alpha_{r} .
\end{aligned}
$$




\subsection{Chern characters and Todd classes}

Let $E$ be a vector bundle of rank $r$ on a variety $X$. We define formally the Chern character and the Todd class of $E$ as follows:

$$
\operatorname{ch}(E)=\sum_{i=1}^{r} \exp \left(\alpha_{i}\right)
$$

and

$$
\operatorname{td}(E)=\prod_{i=1}^{r} \frac{\alpha_{i}}{1-\exp \left(-\alpha_{i}\right)},
$$

where $\alpha_{1}, \ldots, \alpha_{r}$ are the Chern roots of $E$ and the expressions in the $\alpha_{i}$ are understood as formal power series, that is

$$
\exp \left(\alpha_{i}\right)=1+\alpha_{i}+\frac{1}{2} \alpha_{i}^{2}+\frac{1}{6} \alpha_{i}^{3}+\cdots
$$

and

$$
\frac{\alpha_{i}}{1-\exp \left(-\alpha_{i}\right)}=1+\frac{1}{2} \alpha_{i}+\frac{1}{12} \alpha_{i}^{2}+\cdots .
$$

Here are some basic properties of Chern characters and Todd classes:

1. If $E$ and $F$ are two vector bundles, then we have the following formulas:

(a) $\operatorname{ch}(E \otimes F)=\operatorname{ch}(E) \cdot \operatorname{ch}(F)$,

(b) $\operatorname{ch}(E \oplus F)=\operatorname{ch}(E)+\operatorname{ch}(F)$

(c) $\operatorname{td}(E \oplus F)=\operatorname{td}(E) \cdot \operatorname{td}(F)$.

2. The Chern character and the Chern classes of $E$ are related by the following formal identity

$$
\begin{aligned}
\operatorname{ch}(E) & =r+c_{1}+\frac{1}{2}\left(c_{1}^{2}-2 c_{2}\right)+\frac{1}{6}\left(c_{1}^{3}-3 c_{1} c_{2}+3 c_{3}\right)+\cdots \\
& =\sum_{k \geq 0} \frac{p_{k}}{k !}
\end{aligned}
$$

where $c_{i}=c_{i}(E)$ and $p_{k}$ is the determinant of matrix

$$
\left(\begin{array}{ccccc}
c_{1} & 1 & 0 & \cdots & 0 \\
2 c_{2} & c_{1} & 1 & \cdots & 0 \\
\vdots & \vdots & \ddots & \ddots & \vdots \\
\vdots & \vdots & \vdots & \ddots & 1 \\
k c_{k} & c_{k-1} & \cdots & \cdots & c_{1}
\end{array}\right)
$$


3. The Chern character and the Todd class of a vector bundle $E$ are related by the following formula:

$$
\sum_{i=0}^{r}(-1)^{i} \operatorname{ch}\left(\Lambda^{i} E^{\vee}\right)=c_{r}(E) \cdot \operatorname{td}(E)^{-1}
$$

where $r$ is the rank of $E$. This immediately follows using the splitting principle (see Fulton, 1998, Example 3.2.5).

For example, if $X$ is a variety of dimension 3 and $E$ be a vector bundle of rank 2 on $X$. Then we have

$$
\operatorname{ch}(E)=2+c_{1}+\frac{1}{2}\left(c_{1}^{2}-2 c_{2}\right)+\frac{1}{6}\left(c_{1}^{3}-3 c_{1} c_{2}\right)
$$

and

$$
\operatorname{td}(E)=1+\frac{1}{2} c_{1}+\frac{1}{12}\left(c_{1}^{2}+c_{2}\right)+\frac{1}{24} c_{1} c_{2}
$$

where $c_{i}=c_{i}(E)$ be $i$-th Chern class of $E$.

\subsection{Hirzebruch-Riemann-Roch theorem}

We now state the Hirzebruch-Riemann-Roch theorem.

Theorem 2 (Hirzebruch-Riemann-Roch). Let E be a vector bundle on a smooth projective variety $X$. Then we have the following formula:

$$
\chi(X, E)=\int_{X} \operatorname{ch}(E) \cdot \operatorname{td}(X),
$$

where $\chi(X, E)$ is the Euler characteristic of $E$ on $X, \operatorname{td}(X)$ is the Todd class of the tangent bundle on $X$, and $\int_{X} \alpha$ is the degree of the cycle class $\alpha$ in $H^{*}(X, \mathbb{Q})$.

Proof.

See [?, Chapter 4] or [?, Chapter 15].

The Hirzebruch-Riemann-Roch formula theorem gives us an effective method for computing the Euler characteristic of a vector bundle $E$ on a smooth projective variety $X$ in terms of the Chern character of $E$ and the Todd class of the tangent bundle $T_{X}$ on $X$. In the case of projective spaces, the Chern character and the Todd class of a vector bundle on $\mathbb{P}^{n}$ can be considered as polynomials in one variable $h$. Thus the computation can be reduced to polynomials in one variable. For example, let us compute the Chern character of the Tango bundle $T_{n}$ and the Todd class of the tangent bundle on $\mathbb{P}^{n}$.

Lemma 1. Let $n \geq 3$ be an integer. The Chern character of the Tango bundle $T_{n}$ on $\mathbb{P}^{n}$ is computed by the following formula:

$$
\operatorname{ch}\left(T_{n}\right)=(n-1)+\sum_{k=1}^{n} \frac{(-2)^{k}-(-1)^{k}(n+1)}{k !} h^{k},
$$

where $h$ is the class of a hyperplane on $\mathbb{P}^{n}$. 
Proof.

We first determine the total Chern classes of the line bundles $\mathscr{O}_{\mathbb{P}^{n}}(-1)$ and $\mathscr{O}_{\mathbb{P}^{n}}(-2)$,

$$
c\left(\mathscr{O}_{\mathbb{P}^{n}}(-1)\right)=1-h, c\left(\mathscr{O}_{\mathbb{P}^{n}}(-2)\right)=1-2 h .
$$

Then we have

$$
\operatorname{ch}\left(\mathscr{O}_{\mathbb{P}^{n}}(-1)\right)=\sum_{k=0}^{n} \frac{1}{k !}(-h)^{k}, \operatorname{ch}\left(\mathscr{O}_{\mathbb{P}^{n}}(-2)\right)=\sum_{k=0}^{n} \frac{1}{k !}(-2 h)^{k} .
$$

Consider the exact sequence

$$
0 \longrightarrow \mathscr{O}_{\mathbb{P}^{n}}(-2) \longrightarrow \mathscr{O}_{\mathbb{P}^{n}}^{\oplus(n+1)}(-1) \longrightarrow T_{\mathbb{P}^{n}}(-2) \longrightarrow 0,
$$

we have

$$
\begin{aligned}
\operatorname{ch}\left(T_{\mathbb{P}^{n}}(-2)\right) & =(n+1) \operatorname{ch}\left(\mathscr{O}_{\mathbb{P}}(-1)\right)-\operatorname{ch}\left(\mathscr{O}_{\mathbb{P}}(-2)\right) \\
& =(n+1) \sum_{k=0}^{n} \frac{1}{k !}(-h)^{k}-\sum_{k=0}^{n} \frac{1}{k !}(-2 h)^{k} .
\end{aligned}
$$

The exact sequence (1) shows us that

$$
\operatorname{ch}\left(E_{n}\right)=\frac{1}{2} n(n+1)-\operatorname{ch}\left(T_{\mathbb{P}^{n}}(-2)\right) .
$$

Since $F_{2}$ is a trivial bundle, $\operatorname{ch}\left(F_{2}\right)=\operatorname{rank}\left(F_{2}\right)=\frac{1}{2} n(n-1)-n+1$. By the exact sequence (2) we imply that

$$
\begin{aligned}
\operatorname{ch}\left(T_{n}\right) & =\operatorname{ch}\left(E_{n}\right)-\operatorname{ch}\left(F_{2}\right) \\
& =(2 n-1)-\operatorname{ch}\left(T_{\mathbb{P}^{n}}(2)\right) \\
& =(n-1)+\sum_{k=1}^{n} \frac{(-2)^{k}-(-1)^{k}(n+1)}{k !} h^{k} .
\end{aligned}
$$

We have shown that the Chern character of the Tango bundle $T_{n}$ can be considered as a polynomial in one variable $h$. Similarly, we can do the same thing with the Todd classes of tangent bundles on projective spaces. To do so, we need some notations regarding Stirling numbers. We refer to Roman (1984) for more details of these numbers.

The Stiring numbers, denoted by $\left[\begin{array}{l}n \\ k\end{array}\right]$, where $k$ and $n$ are positive integers such that $0 \leq k \leq n$, are defined to be the coefficients of the polynomial

$$
R(x)=\prod_{i=0}^{n-1}(x+i)=\sum_{k=0}^{n}\left[\begin{array}{l}
n \\
k
\end{array}\right] x^{k}
$$


For each non-negative integer $k$, the Stirling polynomial $S_{k}(x)$ (see Roman, 1984, Sect. 4.8.8) is defined by the exponential generating function

$$
\left(\frac{t}{1-e^{-t}}\right)^{x+1}=\sum_{k=0}^{\infty} \frac{S_{k}(x)}{k !} t^{k}
$$

The value of the Stirling polynomial $S_{k}(x)$ at a positive integer $n$ can be calculated by the Stirling number and the binomial coefficient in the following formula

$$
S_{k}(n)=\frac{1}{\left(\begin{array}{l}
n \\
k
\end{array}\right)}\left[\begin{array}{c}
n+1 \\
n-k+1
\end{array}\right] .
$$

With these notations, the Todd class of the tangent bundle on $\mathbb{P}^{n}$ can be expressed as

$$
\operatorname{td}\left(T_{\mathbb{P}^{n}}\right)=\left(\frac{h}{1-e^{-h}}\right)^{n+1}=\sum_{k=0}^{n} \frac{S_{k}(n)}{k !} h^{k} .
$$

\section{PROOF OF THE MAIN RESULT}

By the Hirzebruch-Riemann-Roch theorem, the Euler characteristic of the Tango bundle $T_{n}$ on $\mathbb{P}^{n}$ is computed as follows

$$
\chi\left(\mathbb{P}^{n}, T_{n}\right)=\int_{\mathbb{P}^{n}} \operatorname{ch}\left(T_{n}\right) \cdot \operatorname{td}\left(T_{\mathbb{P}^{n}}\right)=\int_{\mathbb{P}^{n}} \sum_{k=0}^{n} \frac{p_{k}}{k !} h^{k} \cdot \sum_{k=0}^{n} \frac{S_{k}(n)}{k !} h^{k},
$$

where $p_{0}=n-1, p_{k}=(-2)^{k}-(-1)^{k}(n+1), k=1,2, \ldots$ It implies that

$$
\begin{aligned}
\chi\left(\mathbb{P}^{n}, T_{n}\right) & =\sum_{k=0}^{n} \frac{p_{k}}{k !} \cdot \frac{S_{n-k}(n)}{(n-k) !} \\
& =(n-1)+\sum_{k=1}^{n} \frac{p_{k}}{k !} \cdot \frac{S_{n-k}(n)}{(n-k) !} \\
& =(n-1)+\frac{1}{n !} \sum_{k=1}^{n}\left[\begin{array}{l}
n+1 \\
k+1
\end{array}\right] p_{k} .
\end{aligned}
$$

It remains to show that $\sum_{k=1}^{n}\left[\begin{array}{l}n+1 \\ k+1\end{array}\right] p_{k}=n ! n$. By the definition of Stirling numbers, we have

$$
\begin{aligned}
x R(x+1) & =x(x+1)(x+2) \ldots(x+n) \\
& =\sum_{k=0}^{n+1}\left[\begin{array}{c}
n+1 \\
k
\end{array}\right] x^{k} \\
. & =\sum_{k=0}^{n}\left[\begin{array}{l}
n+1 \\
k+1
\end{array}\right] x^{k+1} .
\end{aligned}
$$


Thus

$$
R(x+1)=\sum_{k=0}^{n}\left[\begin{array}{l}
n+1 \\
k+1
\end{array}\right] x^{k}
$$

We can calculate directly $\left[\begin{array}{c}n+1 \\ 1\end{array}\right]=n$ ! and then we have

$$
\sum_{k=1}^{n}\left[\begin{array}{l}
n+1 \\
k+1
\end{array}\right] x^{k}=R(x+1)-n !
$$

Since $R(-1)=R(0)=0$, so we have

$$
\begin{aligned}
\sum_{k=1}^{n}\left[\begin{array}{l}
n+1 \\
k+1
\end{array}\right] p_{k} & =\sum_{k=1}^{n}\left[\begin{array}{l}
n+1 \\
k+1
\end{array}\right](-2)^{k}-(n+1) \sum_{k=1}^{n}\left[\begin{array}{l}
n+1 \\
k+1
\end{array}\right](-1)^{k} \\
& =R(-1)-n !-(n+1)(R(0)-n !) \\
& =n ! n .
\end{aligned}
$$

Theorem 1 is proved as desired.

\section{ACKNOWLEDGEMENT}

This paper is dedicated to Professors Hà Huy Vui and Ta Lê Loi on the special occasion of their birthdays.

\section{REFERENCES}

Dang, T. H. (2014). Intersection theory with applications to the computation of GromovWitten invariants [Doctoral dissertation, Technical University of Kaiserslautern, Germany].

Hirzebruch, F. (1978). Topological methods in algebraic geometry. Springer.

Eisenbud, D., \& Harris, J. (2016). 3264 and all that: A second course in algebraic geometry. Cambridge University Press. https://doi.org/10.1017/CBO9781139062046

Fulton, W. (1998). Intersection theory. Springer-Verlag. https://doi.org/10.1007/978-14612-1700-8

Hartshorne, R. (1979). Algebraic vector bundles on projective spaces: A problem list. To -pology, 18(2), 117-128. https://doi.org/10.1016/0040-9383(79)90030-2

Jaczewski, K., Szurek, M., \& Wisniewski, J. A. (1986). Geometry of the Tango bundle. In H. Kurke \& M. Roczen (Eds.), Proceedings of the Conference on Algebraic Geometry (Berlin, Germany, 1985) (pp. 177-185). B.G. Teubner.

Okonek, C., Schneider, M., \& Spindler, H. (1980). Vector bundles on complex projective spaces, with an appendix by S. I. Gelfand. Birkhäuser (Corrected reprint 2011 by Springer Basel AG). https://doi.org/10.1007/978-3-0348-0151-5

Roman, S. (1984). The umbral calculus. Academic Press. 
Tango, H. (1976). An example of indecomposable vector bundle of rank $n-1$ on $P^{n}$. Journal of Mathematics of Kyoto University, 16(1), 137-141. https://doi.org/ $10.1215 / \mathrm{kjm} / 1250522965$ 